\title{
Signal processing techniques for phase-coded HF-VHF radars
}

\author{
Cesidio Bianchi $\left({ }^{1}\right)$, Umberto Sciacca $\left({ }^{1}\right)$, Achille Zirizzotti $\left({ }^{1}\right)$, Enrico Zuccheretti $\left({ }^{1}\right)$ \\ and Baskaradas James Arokiasamy $\left(^{2}\right)$ \\ ( ${ }^{1}$ Istituto Nazionale di Geofisica e Vulcanologia, Roma, Italy \\ $\left(^{2}\right)$ TRIL fellow, The Abdus Salam International Center for Theoretical Physics, Trieste, Italy
}

\begin{abstract}
HF-VHF radar techniques are easy to employ and commonly used in geophysical applications. They include deep radio soundings, used for probing the ionosphere, stratosphere-mesosphere measurement, weather forecast and radio-glaciology. Fast algorithms and powerful processors facilitate the development of several kinds of low power radars, but the reduction of the transmitted power has to be compensated by on-line processing of an encoded signal to maintain a favorable signal-to-noise ratio suitable for detection. Moreover, radars have to reconstruct return echoes with different travel times due to various origins (multi-path, adjacent objects, etc.). Such needs can be accomplished by means of signal phase coding and one of the most attractive is the reversal phase code. The composite echo signal must be processed to extract the physical information useful for the measurement considered. In this paper some algorithms used for on-line processing of phase-coded signals will be described, both in time and frequency domain.
\end{abstract}

Key words phase-coded radar-pulse compressionsignal integration

\section{Introduction}

The current trend in HF-VHF radars is to simplify the system hardware, reduce transmitted power and develop more powerful techniques of signal processing (Woodmann, 1980; Hunsucker, 1991). The latter is one of the challenging jobs of these radars (phase-coded included), because it consists of a series of tasks, each involving a heavy iterative procedure. With the advent of powerful Digital Signal Processors (DSPs) and very fast PCs, the implementation of these algorithms, that give to HF-VHF radar systems

Mailing address: Dr. Cesidio Bianchi, Istituto Nazionale di Geofisica e Vulcanologia, Via di Vigna Murata 605, 00143 Roma, Italy; e-mail: bianchi@ingv.it the required performances, has become possible. Although these algorithms can be implemented both in time and in frequency domain, working in frequency domain has given faster and more powerful results. Such on-line processing techniques have been used in radar systems developed at INGV (Ariokasamy et al., 2002). The present work illustrates the concepts behind the signal processing techniques implemented in systems like those, though, a more general description and design reference can be found in radar manuals (Skolnik, 1990).

Figure 1 contains the sequence of the most general steps in phase-coded radar signal processing. The essential first step is the quadrature sampling and the Analog-to-Digital (A/D) conversion. In this way the information is available in digital format, ready for the actual processing carried out in the second step. The quadrature sampling is preferred because it allows all the information included in the received signal to be captured for subsequent processing. These two passages are essential for the following detection 
process, to extract relevant information from the received echo (i.e. position, velocity, reflected energy, etc.). The third step is the operations on the processed signal, such as data display or data storage for further analysis (off-line), worked out by a PC.

Working in time domain is easier for some aspects, because the changing of domain introduces two more tasks, the Fourier transform and its inverse. Nonetheless, in frequency domain new processing techniques are possible, so such an approach has become dominant.

Figure 1 is representative enough of the physical units constituting the hardware of the system. The first step is usually implemented by a digital circuitry, which follows an analog receiver providing an amplification and filtering of the received echo (radio frequency). In some cases a good design of the receiver can limit the amount of noise entering the system: the noise produced inside the receiver itself (thermal, due to images, etc.) and those coming from the environment that are not exactly inside the frequency band used

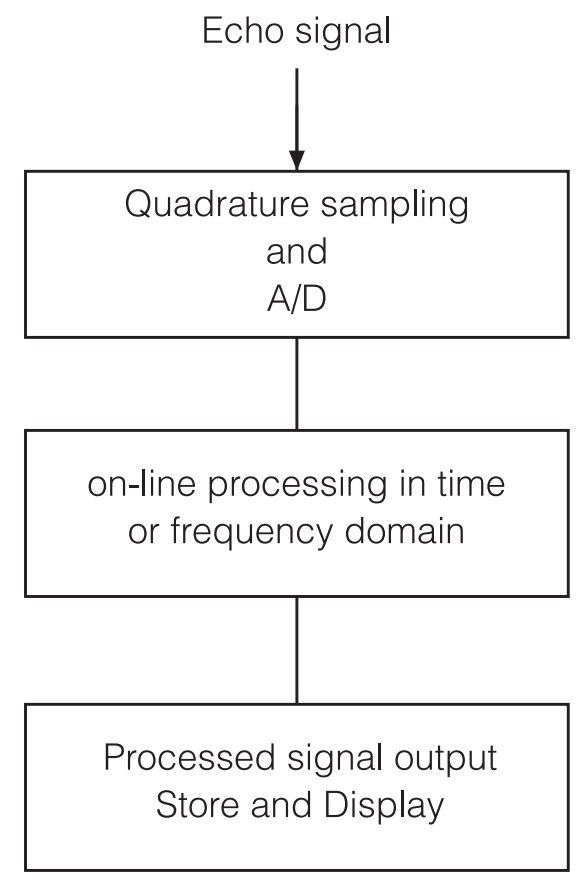

Fig. 1. Signal processing general steps. by the radar system. In other cases it is difficult to discard such external noise, so different methods must be applied to increase the Signalto-Noise ratio $(\mathrm{S} / \mathrm{N})$; they are implemented subsequently.

The signal processing is usually done by a specific DSP, but, because of the calculation power of modern PCs, could be carried also on a general purpose computer. A tradeoff between cost, speed and system requirements has to be made before choosing the type of computing machine.

In the following paragraphs the steps included in the signal processing will be discussed. Before this description, it is important to delineate the methods of phase coding used to improve the radar performance.

\section{Phase coding}

Briefly, the coding of the transmitted signal is adopted to improve the radar resolution increasing the bandwidth. This is necessary both for increasing some features (e.g., maximum distance covered by the system) and for attaining a greater immunity to disturbing sources. To achieve this, the designer cannot use a simple decreasing of the time length of the transmitted pulse without loosing detection capability or increasing the cost of the transmitter. It is well known (Skolnik, 1980) that an increased bandwidth can be attained by means of a proper coding of the signal, now fulfilled in a digital manner. The easiest way to encode the signal is using a particular type of PSK (Phase Shift Keying) that makes the carrier phase change only between two values $\left(0^{\circ}\right.$ and $\left.180^{\circ}\right)$ according to a sequence of binary digits.

Figure 2 depicts what happens when a carrier $\sin (\omega t)$ is multiplied by the sequence of bits $c(t)$ composing the code. The total sequence establishes the length of the pulse. The duration of a single symbol/bit is called «subpulse» and is related to the bandwidth of the encoded signal (the shorter the subpulse, the greater the band). The phase change ( 0 or $\pi$ radians) is obtained at each subpulse $\tau$ or its multiples $n \tau$, while the whole pulse duration is $T$. This linear operation instantaneously gives $m(t)= \pm 1 \cdot \sin (\omega t)$ (that 


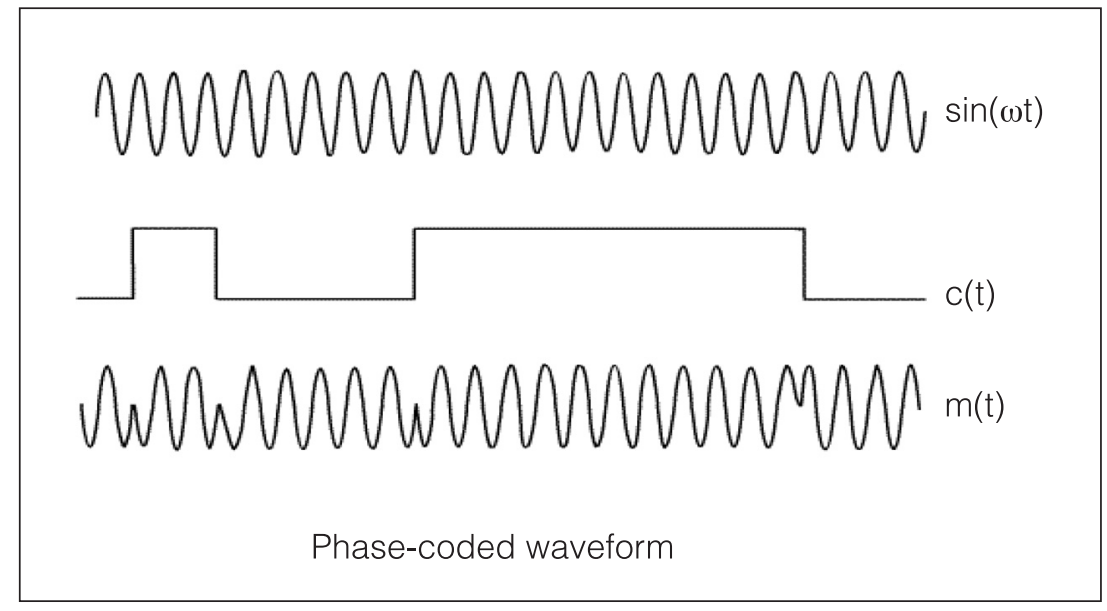

Fig. 2. Bi-phase coding.

is $m(t)=\sin (\omega t)$ if $c(t)=1$ or $m(t)=\sin (\omega t+$ $+\pi)$ in correspondence with $c(t)=-1)$. Using exponential notation

$$
m(t)=c(t) e^{j(\omega t-\phi)}
$$

where $\omega$ is the angular velocity of the wave carrier and $\phi$ is the phase. After the modulation process the signal is amplified, transmitted through the media and a small fraction of energy can be reflected back. The echo signal (replica) $s(t)$, apart from the amplitude, has the same characteristics of $m(t)$ with a noise $N(t)$ superimposed. In practical cases, the echo signal $s(t)$ spans from several tens of $\mathrm{dB}$ above the noise to units of $\mathrm{dB}$ below the noise platform. Because of the multipath propagation the echo signal $s(t)$, which is a composite attenuated and time delayed signal, can be written as

$$
\begin{gathered}
s(t)=\sum_{i} a_{i} c_{i}(t) e^{j\left(\omega t-\phi_{i}\right)} \\
s(t)=\sum_{i} a_{i} m_{i}\left(t-d_{i}\right)
\end{gathered}
$$

where $a_{i}$ is the amplitude of $i$-th multipath component of the signal, and $d_{i}$ is the propagation delay related to the path $i$. The information on the phase $\phi_{i}$ is now contained in $d_{i}$.
Of course a decoding is to be carried out when the echo is received and processed to extract the information it contains; this is usually called correlation and will be described later. Even in case of optimal reception, correlation gives rise to spurious outputs called «sidelobes», that can be seen as a sort of mathematical noise which can interfere with the detection of other echoes.

Many types of codes have been developed, one is the Barker code, characterized by low and equal sidelobes and easy implementation. Codes that reduce sidelobes completely were developed, e.g., the complementary codes (Golay, 1961), using a couple of pulses combined together after correlation. Such codes are harder to implement, because if the combination is not perfect sidelobes appear again. Complementary codes have been used in few radar applications because they rely on a constant radar-target position between code transmission and so they are not particularly suitable for tracking of moving targets. On the contrary, for geophysical application the target distance is in most cases constant. The two mentioned types of codes have been used in the radar systems developed at INGV, giving satisfactory results.

When receiving the echoes, the code should be reconstructed by determination of the phase variations of the carrier. Given that no information on the delay is known, the sampling can- 
not be done in correspondence of the maxima of the carrier, with the risk of losing much of the energy entering the receiver. The best solution is to perform a double sampling, with a couple of values of the received signal for each period of the carrier, the second sample being taken a quarter of a period after the first. In this way, beside sampling for the A/D conversion, a baseband conversion is also performed. After sampling, two streams of data are available, the first usually called the In-phase $(I)$ and the second the Quadrature $(Q)$. This operation leads to discrete-time domain yielding $n$ couple of values, where the signal amplitude and phase are recovered by $A_{n}=\left(I_{n}^{2}+Q_{n}{ }^{2}\right)^{1 / 2}$ and $\phi_{n}=\tan ^{-1}\left(I_{n} / Q_{n}\right)$. A composite baseseband containing all the multipath delays compatible with the sampling window signal remains. This baseband can be expressed mathematically using eq. (2.3) and remembering the change of time from the continuous to the discrete domain

$$
b(n)=\sum_{i} A_{i} c\left(n-d_{i}\right)
$$

$A_{i}$ contains the attenuation information of the single $i$-th multipath echo. Once they have been stored, the actual processing can take place. It is worth remembering that if a multiple echo enters the receiver (due to multipath or to the presence of multiple reflecting structures) the possible superposition of the long received encoded pulses does not necessarily give rise to loss of information, which can be reconstructed after correlation, assuming the time separation between pulses is long enough to be resolved by the system bandwidth.

\section{Time domain processing}

If we choose to perform signal processing in time domain, we can extract the information from the received encoded signal as follows. As stated before, this process is called correlation, it is a sort of weighted moving average of the received signal, the weight being a copy of the encoded transmitted signal stored inside the correlation processor. It is possible to demonstrate that the correlation gives an output shortened in time, or «compressed», reducing the actual duration of the pulse to one subpulse length (neglecting the sidelobes). If the bandwidth of the system is $B W$, the total code length is $T$ and the subpulse is $\tau$ the peak power of the compressed pulse can be increased by a compression factor $M$

$$
M=B W \cdot T=T / \tau
$$

where $B W=1 / \tau$. The position of the maximum and its amplitude give information about distance and reflecting properties of the target. Multiple echoes can be resolved if the time separation between two near received signals is greater than the subpulse length. The actual detection of an echo is obviously determined also by other factors: noise, transmitted power, the attenuation due to the path and the reflecting characteristics of the targets, etc.

In discrete time processing the correlation of the baseband signal $b(n)$, that contains the code sequences (echoes), with $c(n)$, that is the discrete-time version of the function $c(t)$, can be written as

$$
C_{b c}(n)=b(n)^{*} c(n)=\sum_{n=1}^{N} \sum_{m=1}^{M} b(n+m) \cdot c(m)
$$

where $N$ is the number of samples of the acquired baseband temporal segment, $M$ is the code length, $n$ is an index that runs from 1 to $N$ and $m$ is an index that runs from 1 to $M$. Neglecting the side lobes of the correlation, it follows:

$$
C_{b c}(n)=\sum_{i} M A_{i} \delta\left(n-d_{i}\right)
$$

where, $d_{i}$ takes into account all the possible delays (all the replicas compatible with the sampled window), $\delta\left(n-d_{i}\right)$ has value 1 or 0 depending on the lag in the correlation operation. Using a complementary phase code (no side lobes), the relation (3.3) represents the exact result.

Such a time domain process was developed at INGV by means of a DSP. The $I$ and $Q$ streams were combined in order to obtain the real amplitude of the received baseband signal. Then a correlation was performed, both in a system that used the Barker code and in another that used the complementary code (it was a preliminary implementation of the cited AIS-INGV). The processing power gain in deci- 
bels due to this correlation operation is about $G_{C}=20 \log \sqrt{M}(\mathrm{~dB})$. The extraction of the modulus of the received signal was chosen to make the system easy to implement, even though it does not permit a coherent integration, as discussed in a next paragraph.

\section{Frequency domain processing: FFT and correlation}

Figure 3 shows all the passages included in a typical frequency domain process. In the next paragraphs the filtering and integration will be discussed. The present section summarizes some topics on correlation and Fourier transform (Rabiner et al., 1975; Oppenheim et al., 1999).

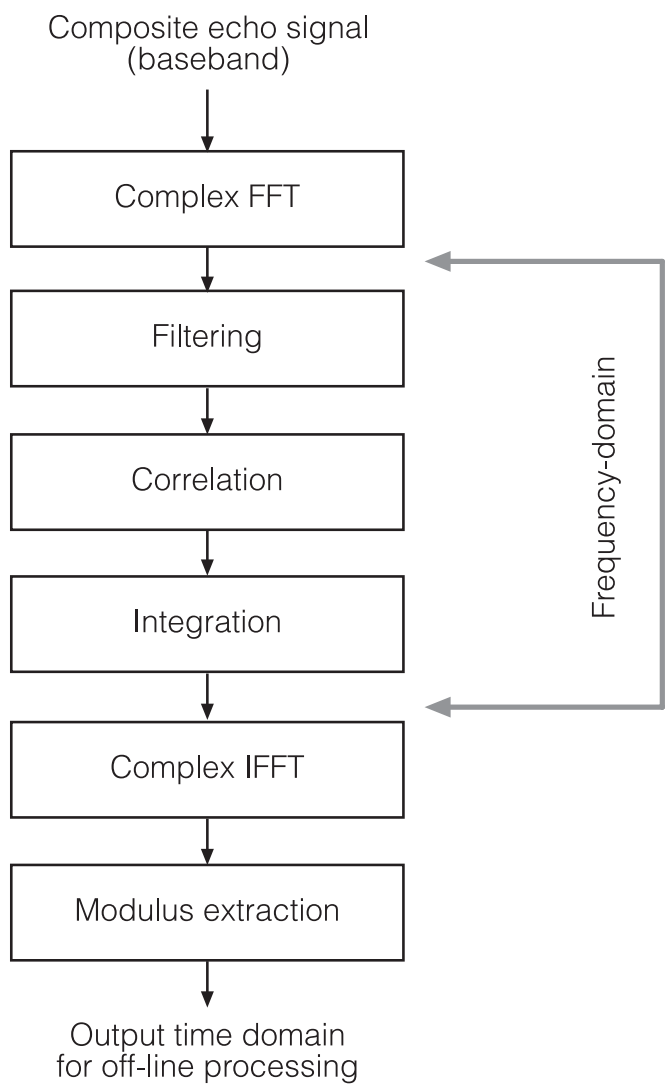

Fig. 3. Frequency domain processing schematics.
It is important to note that the $I$ and $Q$ signals at input are treated as real and imaginary parts of a single complex signal $(I+j Q)$, so the actual algorithm to be used to perform a Fourier transform is a complex FFT (CFFT) and its inverse (ICFFT), having the resulting spectrum different from the real FFT. In addition, all calculations performed in frequency domain take into account a single «complex» signal, even though some processes may act on the real and imaginary part separately. Figure 4 shows an example of CFFT of the acquired data signal.

It is well known from signal theory that correlation can be performed in frequency domain, simply multiplying the spectrum values of the received signal by the ones of the complex conjugate of the transmitted pulse. It follows:

$$
C_{b c}(n)=b(n)^{*} c(n)=B\left(\omega_{n}\right) \cdot C\left(\omega_{n}\right)^{*}
$$

where $B\left(\omega_{n}\right)$ and $C\left(\omega_{n}\right)$ are the time discrete Fourier transform of the baseband echo and the code. This calculation is simpler than the correlation in time domain; it is true that complex numbers have to be multiplied (fig. 5), while in time domain the values were reduced to their modulus, but the price of this simplification was a loss of information, now excluded. It is possible to demonstrate that the loss of computing time due to the transform processes (direct and inverse) can be compensated by the simplification of the correlation; this compensation depends on various factors, mostly the algorithm chosen to perform transformations, the number of samples constituting the signal at the input and the length of the code.

\section{Frequency domain processing: filtering}

Once the spectrum of the received echo is available, it is possible to perform a filtering in different ways. Of course, the classical filtering accomplished by an analog band pass filter is also possible and it was done. A good analog filtering carried out inside the receiver can cut off all the undesired signals entering the system (image frequencies, interfering radio stations, etc.). An analog process is never a perfect one, 


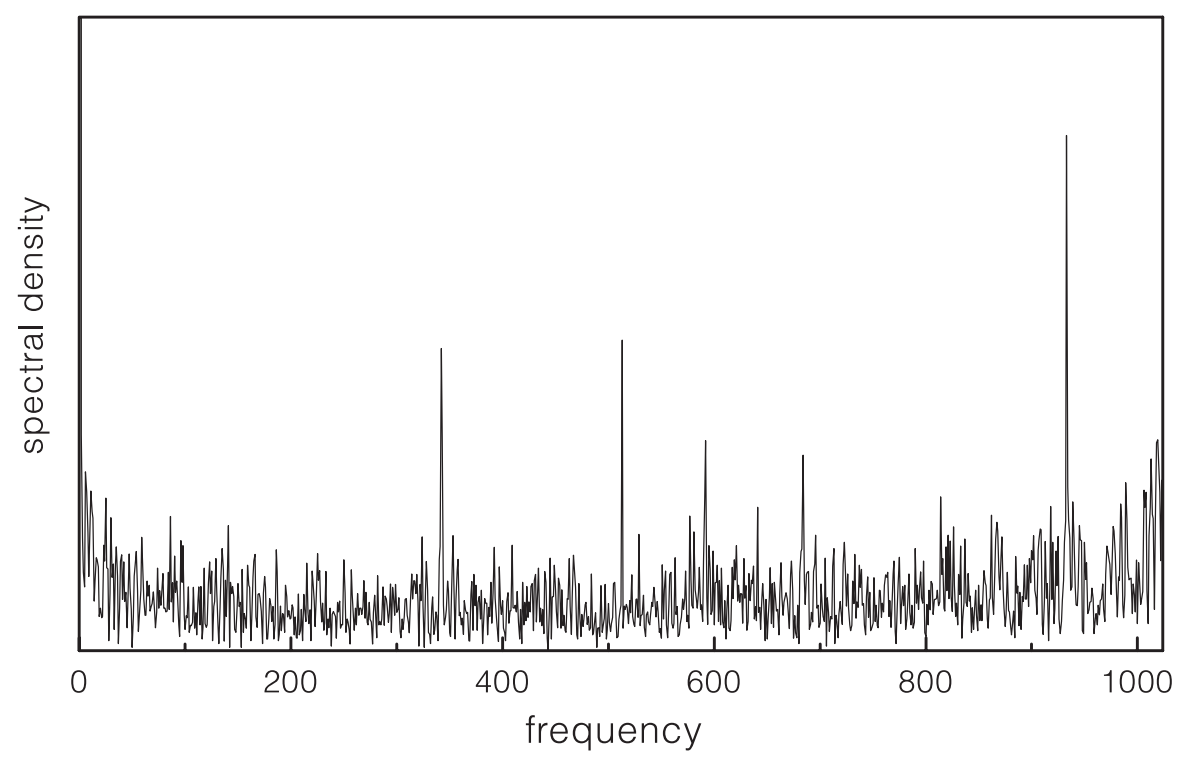

Fig. 4. Spectrum of the complex FFT (CFFT) of the acquired $1024 I$ and $Q$ samples (the $X$-axis in arbitrary units).

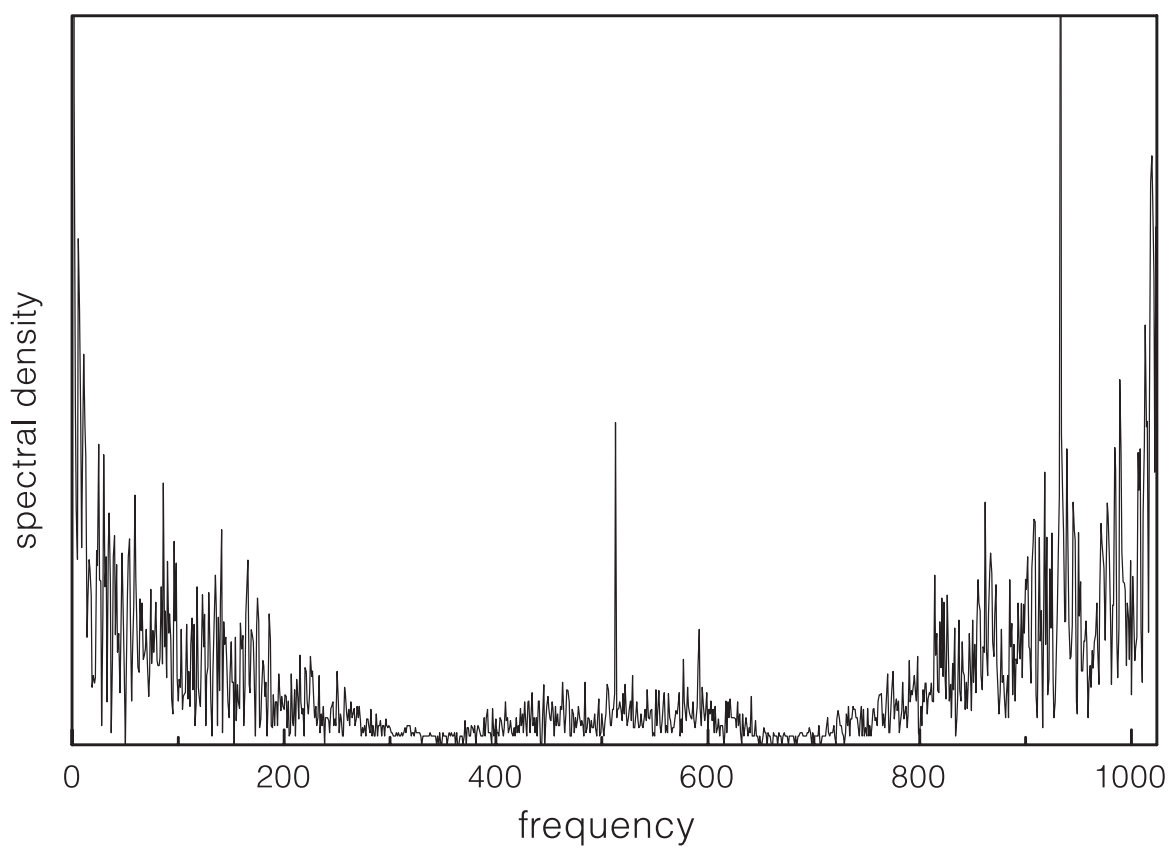

Fig. 5. Correlation in the frequency domain between the CFFT and the spectrum of the code (the $X$-axis in arbitrary units). 
because some residual out-of-band signal can pass through the receiver and appear in the computed spectrum in the DSP. It can be cut off easily simply putting to zero some spectral components, those referring to frequencies beyond a specified limit. It is clear that this kind of filtering cannot resolve hard interference problems, which can be treated only by means of a good design of the receiver.

The real advantage in using a frequency domain filtering is the easy processing of noise lying inside the useful band. In fact, if a strong interfering carrier stays too near to the radar carrier it is not cancelled by a classical filter. The method we used consists in putting to zero (or clipping) all the single spectral lines whose energy overcomes a threshold, assuming that a typical spectrum does not have such «peaks». The threshold value is influenced mostly by the total energy content of the signal entering the processor, but it was determined after empirical trials. This filtering method leads to a slight distortion in the shape of the spectrum, without great consequences. We know it is not the best way to reduce such interfering signals, but it was used for its great ease of implementation.

\section{Frequency domain processing: integration}

The minimum detectable signal can be improved by an integration process (North, 1963), a term indicating a sum of $N$ received signals, acquired after $N$ successive transmissions. The amount of improvement depends on the place in the receiving chain where the integration is carried out. In fact, the noise can also increase its power as a consequence of integration, so the final gain in $\mathrm{S} / \mathrm{N}$ is the result of the combination of the signal and noise integration.

If the integration is done at the end of the receiving chain, i.e. after detection so that the modulus of the signal is extracted, the information on the phase of the signal is lost. In this case, the process is called not-coherent integration; both signal and noise sum their amplitudes in a statistical way (rms), so the gain in the $\mathrm{S} / \mathrm{N}$ (as a power ratio) remains theoretically unchanged. Nonetheless, it is possible to prove that the system increases its performance because of the reduction of the variance of the received signal plus noise. In this way, the probability of detection increases. In some cases, this type of integration is the only possibility, and it is effective enough when the amplitudes of the received signals vary rapidly.

If the phase of the received signal (as a complex function $I+j Q$ ) were known, it could be used to sum all $K$ echoes with the same phase, while the noise would continue changing its phase randomly. In this way, the $\mathrm{S} / \mathrm{N}$ (as a power ratio) would increase by a factor $K$. This process is called «coherent integration». If a sum of the echoes is performed before detection the information on the phase is maintained and, if the phase of the successive echoes does not change, the process can be as effective as a real coherent integration. Equation (4.1), letting $k$ be the index of the integrating echoes (from 1 to $K$ ), becomes

$$
I_{\text {coherent }}=\sum_{k} C_{b c k}(n)=\sum_{k} B_{k}\left(\omega_{n}\right) \cdot C\left(\omega_{n}\right)^{*} .
$$

Obviously this ideal condition never occurs, but it is sufficient for the phase differences between the integrating echoes to remain low. Even great phase differences can increase the $\mathrm{S} / \mathrm{N}$ (not as much as the ideal factor $K$ ), provided they never exceed $\pi / 2$. In order to avoid the phase changing too much, the maximum number of pulses to be integrated should be determined, but this is a very difficult task for the intrinsic variations experienced in the geophysical environment. The processing power gain due to phase coherent integration is about $G_{I}=20 \log \sqrt{K}(\mathrm{~dB})$. In the radar developed (Ariokasamy et al., 2002) the coherent integration was implemented, and the duration of integration was determined empirically. An example of the non integrated signal is given in fig. 6 while in fig. 7 a phase coherent integration process for $K=10$ is shown. A non-coherent integration process can be performed to reduce the noise and signal + noise variance in the post processing operation. 


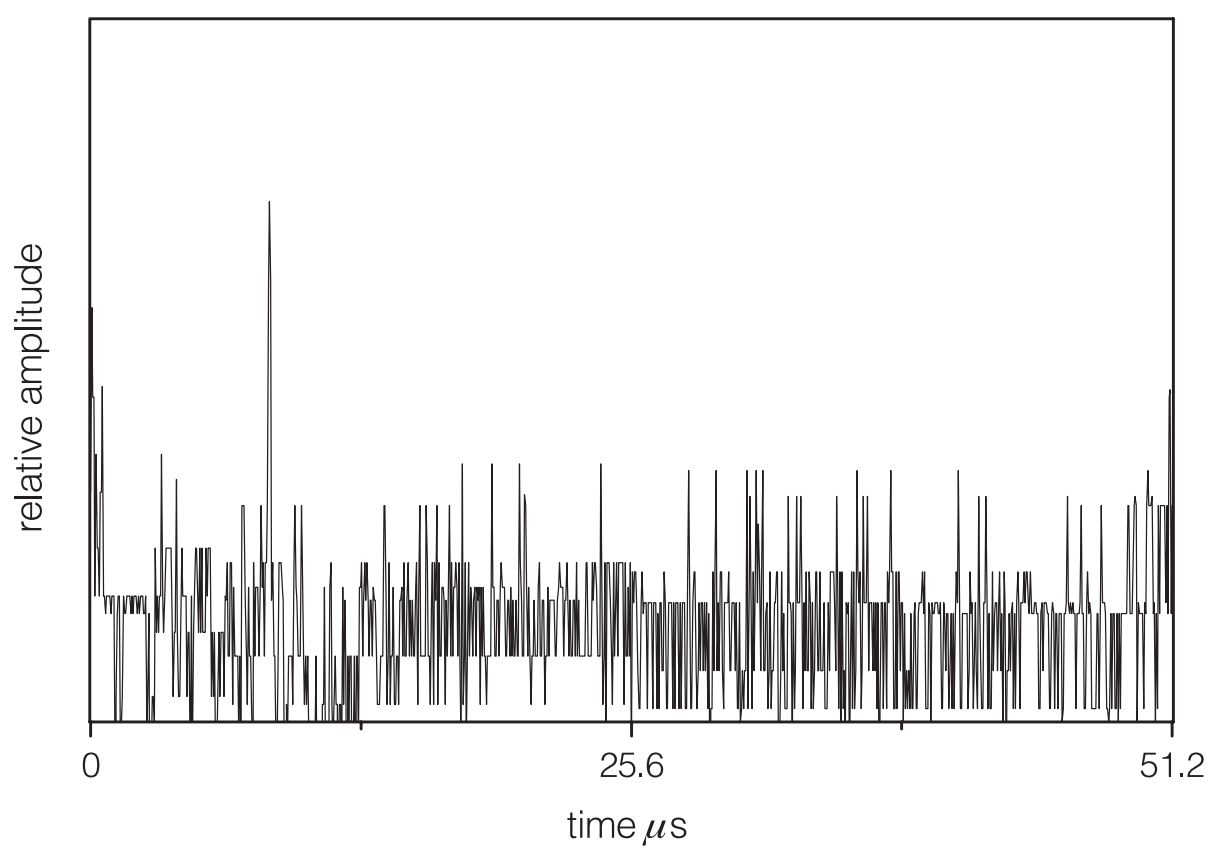

Fig. 6. Inverse complex FFT showing the correlation peak (time delay of the echo).

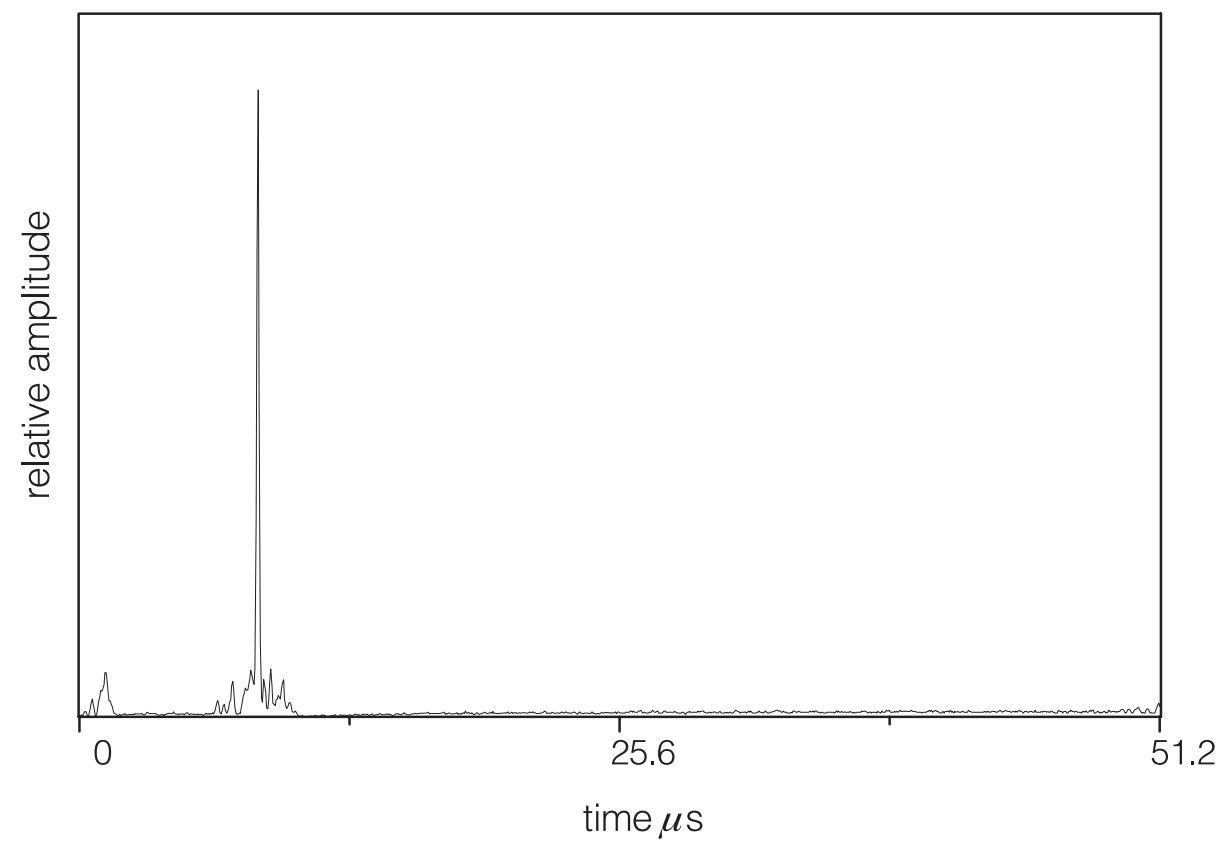

Fig. 7. Resulting peak after ICFFT of 10 iterations of the phase coherent integration process. 


\section{Conclusions}

Low power phase-coded HF-VHF radars useful for geophysical application require some particular processing on the encoded signal to extract physical parameters. The reduced transmitting power (200-500 W), instead of the corresponding power of an envelope radar (about 4000-10000 W), is compensated by an on-line processing gain due to the pulse compression and integration. The described algorithms were implemented at first in a fixed and a floating point DSP board, then also in a PC microprocessor. Those processors were used in a couple of systems developed at INGV: a radar employed for electronic density measurement in the upper atmosphere and a radio echo sounder used for radio glaciology measurements. It was found that knowledge of the physical radio propagative phenomena such as the particular medium and target nature can be useful to extend as long as possible the integration operation without losing the phase coherence. Though the frequency domain signal processing requires more mathematical operations, the speed of the whole process is comparable with the time domain. In fact, operating in frequency domain, the correlation is simply executed by a product of the two spectra and after the integration only a IFFT is required. Another advantage arises implementing a filtering algorithm directly onto the spectrum, clipping and cutting off un- wanted frequency components. These issues, besides allowing less peak transmitted power, also improve the overall signal-to-noise ratio of the employed radar systems for geophysical applications.

\section{REFERENCES}

Arokiasamy, B.J., C. Bianchi, U. Sciacca, G. Tutone and E. ZUCCHERETTI (2002): The new AIS-INGV digital ionosonde design report, INGV Internal Technical Report n. 12.

GolAy, M.J.E. (1961): Complementary series, IRE Transaction on Information Theory, IT-7, 82-87.

HUNSUCKER, R.D. (1991): Radio technique for probing the terrestrial ionosphere, Physics and Chemistry in Space, edited by M.C.E. HubER, L.J. LANZEROTTI and D. STOFFLER (Springer-Verlag, Berlin Heidelberg), vol. 22.

NORTH, D.O. (1963): An analysis of the factors which determine the signal/noise discrimination in pulsed carrier systems, Proc. IEEE, 51 (7), 1015-1028.

OpPenheIM, V., A. Schafer, W. RONALD and J. R. BucK, (1999): Discrete-Time Signal Processing (Englewood Cliffs, New Jersey, Prentice Hall), 2nd edition, pp. 870.

RABINER, L.R. and B. GOLD (1975): Theory and Application of Digital Signal Processing (Englewood Cliffs, New Jersey, Prentice Hall), pp. 762.

SKOLNIK, M.I. (1980): Introduction to Radar Systems (Mc Graw-Hill, New York), 2nd edition, pp. 442.

SkolNIK, M.I. (1990): Radar Handbook (Mc Graw-Hill, New York), 2nd edition, pp. 1200.

WoOdMAN, R.F. (1980): High altitude resolution stratospheric measurements with the Arecibo $430 \mathrm{MHz}$ radar, Radio Sci., 15, 417-422.

(received February 5, 2003; accepted July 11, 2003) 